\title{
COMBINING JPEG STEGANOGRAPHY AND SUBSTITUTION ENCRYPTION FOR SECURE DATA COMMUNICATION
}

\author{
Shamim Ahmed Laskar ${ }^{1}$ and Kattamanchi Hemachandran ${ }^{2}$ \\ Department of Computer Science \\ Assam University, Silchar, Assam, India \\ ${ }^{1}$ shamim.auserediffmail.com, ${ }^{2}$ khchandran@rediffmail.com
}

\begin{abstract}
The Internet is a method of communication to distribute information to the masses. Digital image are excellent carriers for hidden information. Steganography and cryptography are technologies that are used for secret and secured communications. In both the methods, secret message is exchanged between two groups, sender and receiver. The main purpose in cryptography is to make massage concept unintelligible, while steganography aims to hide secret message. We propose a method of combining steganography, cryptography for secret data communication. In this paper, we propose a high-performance JPEG steganography along with a substitution encryption methodology. This approach uses the discrete cosine transform $(D C T)$ technique which used in the frequency domain for hiding data within image. It is very difficult to detect hidden message in frequency domain and for this reason we use steganography based on DCT. From the experimental results, we obtain that the proposed method has a larger message capacity. Experimental results show that the correlation and entropy values of the image with encrypted data before the insertion are similar to the values of correlation and entropy after the insertion thus reduces the chance of the confidential message being detected and enables secret communication. The image that contains the hidden data will be used by the receiver to reconstruct the same secret message after extracting it. The effectiveness of the proposed method has been estimated by computing Mean square error (MSE) and Peak Signal to Noise Ratio (PSNR). Experimental results show the method provides high security and the information is safe from various attacks.
\end{abstract}

\section{Keywords}

Steganography, Cryptography, plaintext, encryption, decryption, ciphertext, substitution cipher, discrete cosine transform, JPEG, quantization, Mean square error and Peak Signal to Noise Ratio.

David C. Wyld, et al. (Eds): CCSEA, SEA, CLOUD, DKMP, CS \& IT 05, pp. 149-160, 2012.

(C) CS \& IT-CSCP 2012

DOI : $10.5121 /$ csit.2012.2216 


\section{INTRODUCTION}

In today's world the data is the heart of computer communication and global economy. To ensure the security of the data so that it does not go to unintended destination, the concept of data hiding has attracted people to come up with creative solutions to protect data from falling into wrong hands [1]. Digital data can be delivered over computer networks from one place to another without any errors and often without interference. The distribution of digital media raised a concern over the years as the data are attacked and manipulated by unauthorized person [2]. Digital data can be copied without any loss in quality and content. Thus it poses a big problem for the security of data and protection of intellectual property rights of copyright owners [3]. The Internet provides an increasingly broad band of communication as a means to distribute information to the masses. As a result of spreading the Internet all around the world, motivation of hiding secret message in different multimedia and secure communication via Internet is increased [5]. Techniques for information hiding have become increasingly more sophisticated and widespread. Such information includes text, images, and audio to convey ideas for mass communication which provide excellent carriers for hidden information. Due to the growth of data communication over computer network, the security of information has become a major concern [4]. Therefore, the confidentiality and data integrity are required to be protected against unauthorized access and use. Steganography and cryptography are the two different information hiding and protecting techniques, where we transform the message so as to make it meaning obscure to a malicious people trying to intercept [8].

Steganography is a data hiding technique aiming to transmit a message on a channel, where some other kind of information is already being transmitted [6]. The goal of steganography is to hide messages inside other "harmless" digital media in a way that does not allow any person to even detect the secret message present [4]. The information that is to be hidden is encoded in a manner such that the very existence of the information is concealed. The main goal of steganography is to communicate securely in such a way as to avoid drawing suspicion to the transmission of a hidden data [7]. Cryptography, on the other hand obscures the content of the message, so it cannot be understood where the existence of the message itself is not disguised [4]. Cryptography hides the contents of a secret message from a unauthorized person but the content of the message is visible [12]. In cryptography, the structure of a message is scrambled in such a way as to make it meaningless and unintelligible manner but it makes no attempt to disguise or hide the encoded message. Basically, cryptography offers the ability of transmitting information between persons in a way that prevents a third party from reading it [11].

Steganography does not alter the structure of the secret message, but hides it inside a medium so it cannot be seen [7]. In other words, steganography prevents an unintended recipient from suspecting that the data exists. In addition, the security of the steganography system relies on secrecy of the data encoding system [1]. Once the encoding system is known, the steganography system is defeated. While cryptography protects messages from being captured by unauthorized parties, steganography techniques enable concealment of the fact that a message is being sent so that it could not be felt that a message is embedded into digital media. Steganography is the invisible communication between the sender and the receiver [14]. In Steganography, only the sender and the receiver know the existence of the message, whereas in cryptography the existence of the encrypted message is visible to the world [13]. Due to this, Steganography removes the unwanted attention coming to the media in which the message is hidden. Cryptographic methods try to protect the content of a message, while Steganography uses methods that would hide both the message as well as the content [30]. 
Although, the two techniques are different in their way of data hiding but they are in fact complementary techniques. No matter how strong algorithm, if an encrypted message is discovered, it will be subject to cryptanalysis [31]. Likewise, no matter how well a message is concealed inside a digital media there is possibility of the hidden message to be discovered. By combining Steganography and Cryptography we can achieve better security by concealing the existence of an encrypted message [9]. It is possible to combine the techniques by encrypting message using cryptography and then hiding the encrypted message using steganography [8]. The resulting stego-object can be transmitted without revealing that secret information is being exchanged. Furthermore, even if an attacker were to defeat the steganographic technique and detect the message from the stego-object, he at first have to decode the message from digital media and then he would still require the cryptographic decoding algorithm to decipher the encrypted message [10].

\section{IMAGE BASED STEGANOGRAPHY}

It has been observed that all digital file formats can be used hiding data using steganography, but the formats those have a high degree of redundancy present in them are more suitable. The redundant bits of an object are those bits that can be altered without the alteration being detected easily $[4,16]$. As digital images contains large amount of redundant bits, they are the most popular cover objects for steganography. This is relatively easy because an image, being an array of pixels, typically contains an enormous amount of redundant information [6]. To a computer, an image is a collection of numbers that constitute different light intensities in different areas of the image. This numeric representation forms a grid and the individual points are referred to as pixels [32]. These pixels make up the image's raster data. Image based steganography is about exploiting the limited powers of the human visual system (HVS) [5, 28]. There are plenty of ways to hide messages within images. The security of stego-images depends entirely on their ability to go unnoticed [5].

When working with digital images, the images seems to be too large to be transmitted over the Internet. Thus in orders to display an image in a reasonable amount of time, techniques are used to reduce the image's file size [24]. These techniques make use of mathematical formulas to analyze and reduce image data, resulting in smaller file sizes and this process is called compression [7]. Steganographic technique is equally important as the choice of the cover image thus compression plays a very important role in image based steganography methods. Current image formats can be divided into two broad categories, lossy and lossless. Both methods save storage space but have different results. Lossless compression reconstructs the original message exactly and thus it is preferred when the original information must remain intact $[15,16]$. Lossless images are more suitable for embedding, since the integrity of the image data is preserved. However, they do not have the high compression ratio that lossy formats do. Lossy compression, on the other hand, saves space but may not maintain the original image's integrity. The plus side of lossy images, in particular JPEG, is that it achieves extremely high compression, while maintaining fairly good quality $[16,17]$. Earlier it was thought that steganography would not be possible using JPEG images, since they use lossy compression [4] which results in parts of the image data being altered. One of the major characteristics of steganography is the fact that information is hidden in the redundant bits of an object and since redundant bits are left out when using JPEG it was feared that the hidden message would be destroyed [14]. However, the properties of the compression algorithm have been exploited in order to develop a steganographic 
algorithm for JPEGs [18]. One of these properties of JPEG is exploited to make the changes to the image invisible to the human eye [20]. This technique, whilst crude, hides a large volume of information inside the image [6]. Once implemented, it is not necessarily perceptible to a human eye that the image has been changed. Lossy compression is preferred in image based steganography because it achieve higher compression compared to lossless compression and thus it is much more secure and have less chances of detection that that of lossless. Steganography not only deals with embedding the secret data inside the digital image but also the receiver to whom the message is intended must know the method used and would be able to retrieve the message successfully without drawing the attention of a third party that a secret communication is occurring.

\section{Proposed techniQue}

In this paper we propose a method for hiding large volumes of data in digital images by combining cryptography and steganography while incurring minimal perceptual degradation in terms of human visual interpretation and to solve the problem of unauthorized data access. Steganography also can be implemented to cryptographic data so that it increases the security of this data $[8,9]$. In this method we first encrypt a message using substitution cipher method and then embed the encrypted message inside a JPEG image using DCT in frequency domain. A substitution cipher is one in which each character in the plaintext is substituted for another character in the ciphertext [31]. Thus the original message that is represented in such a form that is not meaningful to the third party. As we know that the JPEG compression is based on the discrete cosine transform (DCT) and reduces the visual redundancy to achieve good compression performance [17]. Thus it is very difficult to detect hidden message in frequency domain and for this reason we use transformation like DCT in our proposed technique. Therefore, the embedding capacity provided by JPEG steganography is less prone to detection. The combination of these two methods will enhance the security of the data embedded. This combinational methodology will satisfy the requirements such as capacity, security and robustness for secure data transmission over an open channel [10]. The resulting stego-image can be transmitted without revealing that secret information is being exchanged. Furthermore, even if an attacker were to defeat the steganographic technique to detect the message from the stego-object, he would still require the cryptographic decoding method to decipher the encrypted message [8]. The intended receiver should be able to recover the embedded data successfully, without any errors. The proposed methods can be employed for applications that require high-volume embedding with robustness against attacks.

\subsection{Encrypting message}

The proposed method uses a cryptographic system based on classical encryption technique i.e. substitution method, A substitution cipher substitutes one piece of information for another. In substitution cipher each character in the plaintext is substituted for another character in the ciphertext [13]. Such ciphertext could be transmitted across a network or stored within a file system with the disguise serving to provide confidentiality [25]. Instead of changing the order of characters as in transposition cipher, the substitution cipher replaces the character of the message with other character so as to make the message unintelligible [26]. In substitution cipher, the algorithm is to offset the alphabet and the key is the number of characters to offset it [31]. The receiver inverts the substitution on the ciphertext to recover the plaintext [25]. For example, if we encrypt the word "MESSAGE" by shifting 16 places, then we have "MESSAGE" encrypts as 
"CUIIQWU". To allow someone else to read the ciphertext, we tell the recipient that the key is 16. Now if we suppose A (sender) wants to send B (recipient) the plaintext message $M$ over the insecure communication line, $\mathrm{A}$ encrypts $\mathrm{M}$ by computing the ciphertext $\mathrm{C}=\mathrm{E}(\mathrm{K}, \mathrm{M})$ and sends $\mathrm{C}$ to $\mathrm{B}$. Upon receipt, $\mathrm{B}$ decrypts $\mathrm{C}$ by computing $\mathrm{M}=\mathrm{D}(\mathrm{K}, \mathrm{C})$. The adversary may know $\mathrm{E}$ and $\mathrm{D}$ are the encryption and decryption algorithms respectively which are being used in the process.

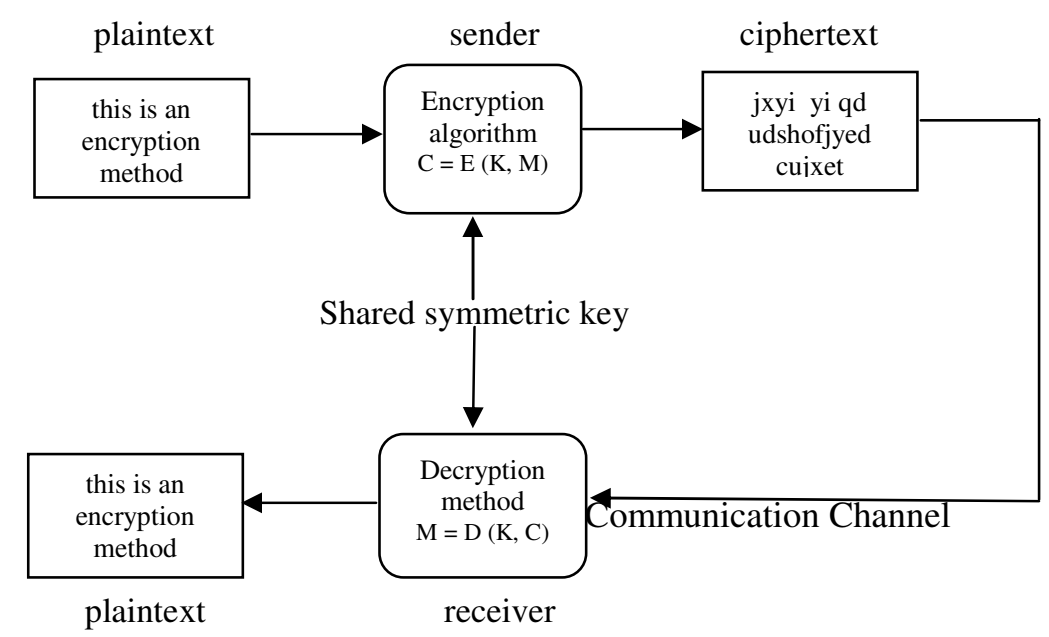

Fig 1: Symmetric encryption system

- Plaintext: It is the original message which is to be transmitted. Formally, it can be written as $\mathrm{M}=<\mathrm{m} 1, \mathrm{~m} 2, \ldots, \mathrm{mn}>$ to denote the plaintext. For example, $\mathrm{M}=<\mathrm{t}, \mathrm{h}, \mathrm{i}, \mathrm{s}, \mathrm{i}, \mathrm{s}, \mathrm{a}, \mathrm{n}$, ,e,n,c,r,y,p,t,i,o,n, ,m,e,t,h,o,d >.

- Ciphertext: It is the translated or encrypted message, which can be denoted as $\mathrm{C}=<\mathrm{c} 1$, $\mathrm{c} 2, \ldots, \mathrm{cm}>$. Thus, the ciphertext $\mathrm{C}=<\mathrm{j}, \mathrm{x}, \mathrm{y}, \mathrm{i}, \mathrm{y}, \mathrm{i}, \mathrm{,q}, \mathrm{d}, \mathrm{,u}, \mathrm{d}, \mathrm{s}, \mathrm{h}, \mathrm{o}, \mathrm{f}, \mathrm{j}, \mathrm{y}, \mathrm{e}, \mathrm{d}, \mathrm{,c}, \mathrm{u}, \mathrm{j}, \mathrm{x}, \mathrm{e}, \mathrm{t}>$.

- Encryption: The process of encoding a message so that its meaning is not obvious; the transformation from plaintext to ciphertext. The encryption is denoted using $\mathrm{C}=\mathrm{E}(\mathrm{M})$, where $\mathrm{C}$ is the ciphertext and $\mathrm{M}$ is the plaintext and $\mathrm{E}$ is the encryption method. In the present substitution method, $\mathrm{C}=\mathrm{E}(\mathrm{M})=(\mathrm{M}+16) \bmod (26)$; in general, $\mathrm{C}=\mathrm{E}(\mathrm{M})=(\mathrm{M}$ $+\mathrm{K}) \bmod (26)$, where $\mathrm{K}$ is the key.

- Decryption: It is the reverse process of encryption; the transformation from ciphertext to plaintext, formally denoted as $M=D(C)$. In the present substitution method, $M=D(C)=$ $(C-16) \bmod (26)$; in the general, $M=D(C)=(C-K) \bmod (26)$, where $K$ is the key.

- Key: Key is something that is set based on the agreement between the sender and the recipient. In the present encryption method the key tells how much to shift. It is an input to the encryption and decryption algorithm [31]. The encryption algorithm will produce a different ciphertext depending on the specific key being used. The corresponding key is needed to decrypt the ciphertext to plaintext [27]. A key gives us flexibility in using an encryption algorithm and provides additional security [19]. If the encryption algorithm is known to the third party still the messages can be kept secret because the attacker does 
not know the key value used in the encryption process. When same key used for encryption and decryption as shown in Fig. 1, they are called symmetric key, or secret key [19]. The encryption (symmetric-key encryption or secret- key encryption) process can be denoted as $\mathrm{C}=\mathrm{E}(\mathrm{K}, \mathrm{M})$; and the decryption process is denoted as $\mathrm{M}=\mathrm{D}(\mathrm{K}, \mathrm{C})$. The cryptosystem is called symmetric cryptosystem and needs to satisfy $\mathrm{M}=\mathrm{D}(\mathrm{K}, \mathrm{E}(\mathrm{K}, \mathrm{M}))$.

\subsection{Embedding the encrypted message in image file}

Images are the most popular cover objects for steganography because of large amount of redundant bits which are suitable for data transmission on the Internet [24]. An example of an image format that uses this compression technique is JPEG (Joint Photographic Experts Group) [17]. JPEG is the most popular image file format on the Internet and the image sizes are small because of the compression, thus making it the least suspicious algorithm to use. The JPEG format uses a discrete cosine transform to image content transformation. DCT is a widely used tool for frequency transformation [23]. In JPEG image format, message bits are inserted in the DCT (Discrete Cosine Transforms) coefficients. The quantized DCT coefficients formed in the JPEG compression are used to embed the secret bits.

Embedding process is discussed in four phases as follows:

- $\quad$ Phase One: Divide the Image

To compress an image into JPEG format, the RGB colour representation is first converted to a YUV representation space and break up each colour plane into $8 \times 8$ blocks of pixels [18, 21]. In this representation the $\mathrm{Y}$ component corresponds to the luminance (or brightness) and the $\mathrm{U}$ and $\mathrm{V}$ components stand for chrominance (or colour) [23]. The chrominance coefficients of an image are determined by a 2D grid that has blue to yellow on one axis, and red to green on another [22]. According to research the human eye is more sensitive to changes in the brightness (luminance) of a pixel than to changes in its colour. This means that it is possible to remove a lot of colour information from an image without losing a great deal of quality [17]. This fact is exploited by the JPEG compression by down sampling the colour data to reduce the size of the file. The colour components $(\mathrm{U}$ and $\mathrm{V}$ ) are halved in horizontal and vertical directions, thus decreasing the file size by a factor of 2 .

- Phase Two: Conversion to the Frequency Domain

The next step is the actual transformation of the image. The DCT transforms [20] a signal from an image representation into a frequency representation, by grouping the pixels into $8 \times 8$ pixel blocks and transforming the pixel blocks into 64 DCT coefficients each [22]. A modification of a single DCT coefficient will affect all 64 image pixels in that block.

Discrete cosine transformations (DCT) are used by the JPEG compression algorithm to transform successive $8 \times 8$ pixel blocks of the image, into 64 DCT coefficients each [24]. Each DCT coefficient $F(u, v)$ of an $8 \times 8$ block of image pixels $f(x, y)$ is given by:

$$
F(u, v)=\frac{1}{4} C(u) C(v)\left[\sum_{x=0}^{7} \sum_{y=0}^{7} f(x, y) * \cos \frac{(2 x+1) u \pi}{16} \cos \frac{(2 y+1) v \pi}{16}\right]
$$




$$
\begin{array}{r}
\text { where } C(\mathrm{u})=1 / \sqrt{2} \text { when } \mathrm{u}=0 \text { and } \mathrm{C}(\mathrm{u})=1 \text { otherwise. } \\
\mathrm{C}(\mathrm{v})=1 / \sqrt{2} \text { when } \mathrm{v}=0 \text { and } \mathrm{C}(\mathrm{v})=1 \text { otherwise. }
\end{array}
$$

\section{- Phase Three: Quantization}

Having the data in the frequency domain allows the algorithm to discard the least significant parts of the image. The JPEG algorithm does this by dividing each cosine coefficient in the data matrix by some predetermined constant, and then rounding up or down to the closest integer value [21]. The constant values that are used in the division may be arbitrary. The next step is the quantization [17] phase of the compression. The aim is to quantize the values that represent the image after transforming values to frequencies [22]. Quantization is the process of taking the 64 DCT coefficients and dividing them individually against a predetermined set of values and then rounding the results to the nearest real number value $[6,18]$. The human eye is fairly good at spotting small differences in brightness over a relatively large area, but not so good as to distinguish between different strengths in high frequency brightness [23]. This means that the strength of higher frequencies can be diminished, without changing the appearance of the image. JPEG does this by dividing all the values in a block by a quantization coefficient [22].

After calculating the coefficients, the following quantizing operation is performed:

$$
\mathrm{F}^{\mathrm{Q}}(\mathrm{u}, \mathrm{v})=\left\lfloor\frac{\mathrm{F}(\mathrm{u}, \mathrm{v})}{\mathrm{Q}(\mathrm{u}, \mathrm{v})}\right\rfloor
$$

where $\mathrm{Q}(\mathrm{u}, \mathrm{v})$ is a 64-element quantization table.

After quantization the encrypted message bits are embedded into the DCT coefficients. DCT coefficients transform a signal or image from the spatial domain to the frequency domain. DCT is used in image steganography is broken into $8 \times 8$ blocks of pixels. Working from left to right, top to bottom, the DCT is applied to each block [18]. Each block is compressed through quantization table to scale the DCT coefficients and encrypted message is embedded in quantized DCT coefficients. The selected coefficients after quantization are ordered by magnitude and then modified by the corresponding bit in the message stream. The quantization step is lossy because of the rounding error [22]. The quantized coefficients are then passed to the entropy encoding step to form the compressed code.

- Phase Four: Entropy Coding

After quantization, zig-zag type motion is performed so that similar frequencies are grouped together. Zigzag ordered encoding collects the high frequency quantized values into long strings of zeros [21]. In zigzag small unimportant coefficients are rounded to 0 while larger ones lose some of their precision [18]. To perform a zigzag encoding on a block, the algorithm starts at the discrete cosine value and begins winding its way down the matrix. This converts an $8 \times 8$ table into a 1 x 64 vector. The results are rounded to integer values and the coefficients are encoded using Huffman coding to further reduce the size [17]. All of the values in each block are encoded in this zigzag order except for the discrete cosine value. Huffman coding scans the data being written and assigns fewer bits to frequently occurring data, and more bits to infrequently occurring 
data [23]. Discrete cosine values use delta encoding, which means that each discrete cosine value is compared to the previous value, in zigzag order. The size field for discrete cosine values is included in the Huffman coding for the other size values, so that JPEG can achieve even higher compression of the data.

Thus it is important to recognize that the JPEG compression algorithm is actually divided into lossy and lossless stages [7]. The DCT and the quantization phase form part of the lossy stage, while the Huffman encoding used to further compress the data is lossless. Steganography can take place between these two stages [14].Using the same principles of insertion the encrypted message can be embedded into DCT coefficients before applying the Huffman encoding [17]. By embedding the information at this stage, in the transform domain, it is extremely difficult to detect, since it is not in the visual domain [18]. Transform embedding in which a message is embedded by modifying coefficients of the cover image (result is called the stego-image).Transform embedding methods are found to be in general more robust than other embedding methods which are susceptible to image-processing type of attacks [23].

\section{EXPERIMENTAl AnAlysis}

In the proposed method we first encrypt the plaintext to generate the ciphertext using substitution cipher method, and then the ciphertext is embedded inside the JPG image file using DCT technique. The generated stego-image is sent over to the intended recipient. Once the recipient receives the stego-image, the ciphertext is extracted from it by reversing the logic that was used to embed it in the first place. The ciphertext is decrypted using the substitution cipher method to get back the original plaintext. The messages were successfully embedded into the cover images.

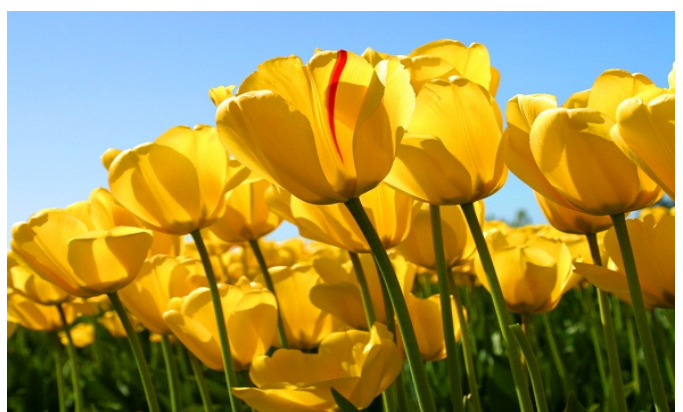

a

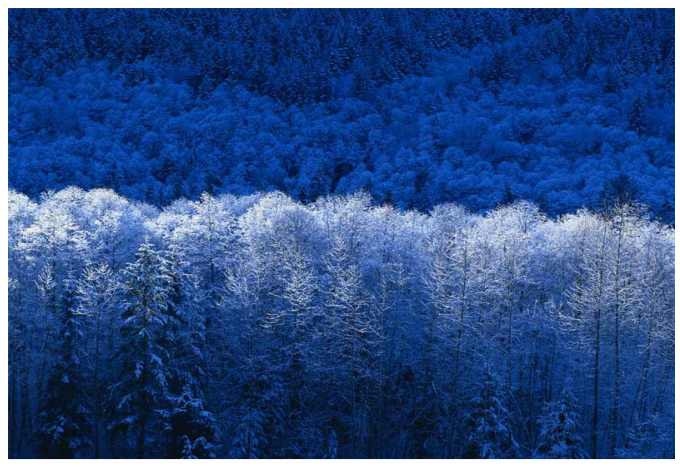

c

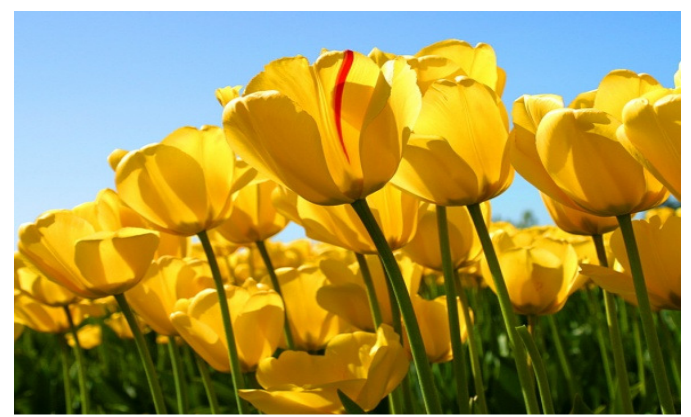

b

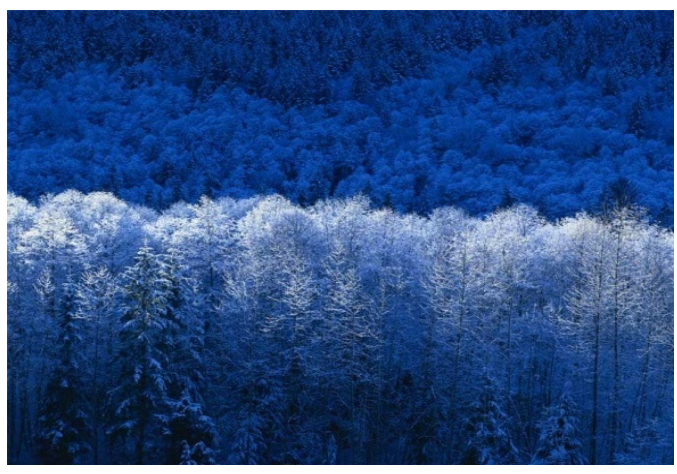

d 


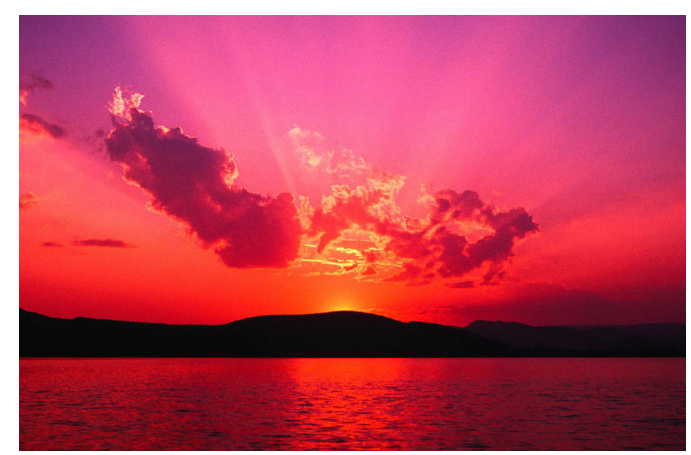

e

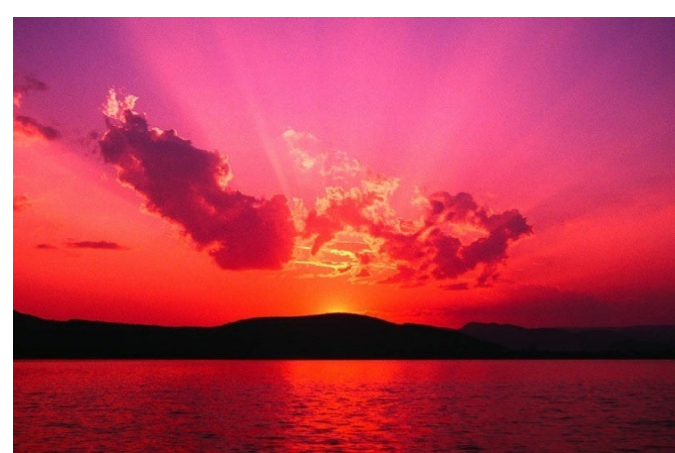

f

Fig. 2. tulips (a) Original image (b) stego image winter (c) Original image (d) stego image, sunset (e) Original image (f) stego image.

The messages were also extracted successfully. In the experiment it is observed that the human visual system (HVS) cannot distinguish the cover-image and stego image [28] the complexity of the image is not disturbed as shown in figure 2 (a) and (b), (c) and (d), (e) and (f). Distortion analysis of stego images is carried out by studying distortion / similarity statistically. Distortion between two different images is measured by considering Mean Square Error (MSE), and PSNR (peak signal to noise ratio) [29].

Usually, the invisibility of the hidden message is measured in terms of the Peak Signal-to-Noise Ratio [28]. To analyze the quality of the embedded texture image, with respect to the original, the measure of PSNR has been employed [29]:

$$
P S N R=10 \log _{10} \frac{255^{2}}{M S E}
$$

where mean square error (MSE) is a measure used to quantify the difference between the cover image I and the stego (distorted) image I' [28]. If the image has a size of $\mathrm{M} * \mathrm{~N}$ then

$$
M S E=\frac{1}{M \times N} \sum_{i=1}^{M} \sum_{j=1}^{N}\left[I(i, j)-I^{\prime}(i, j)\right]^{2}
$$

TABLE 1. MSE and PSNR values for the Original and Stego images

\begin{tabular}{|l|l|l|l|l|l|}
\hline $\begin{array}{l}\text { Cover } \\
\text { image }\end{array}$ & $\begin{array}{l}\text { Stego } \\
\text { Image }\end{array}$ & No. of bytes embedded & $\begin{array}{l}\text { MSE } \\
\text { \% }\end{array}$ & $\begin{array}{l}\text { PSNR } \\
\text { (dB) }\end{array}$ & $\begin{array}{l}\text { No. of bytes } \\
\text { extracted }\end{array}$ \\
\hline tulips & steg_tulips & 2213 bytes & 6.22 & 40.19 & 2213 bytes \\
\hline winter & steg_winter & 1628 bytes & 3.54 & 42.63 & 1628 bytes \\
\hline sunset & steg_sunset & 1323 bytes & 1.71 & 45.79 & 1323 bytes \\
\hline
\end{tabular}


Generally speaking, when the payload increases, the MSE will increase, and this will affect the PSNR inversely [23]. So, from trade-off it was found that MSE decrease causes PSNR increase and vice-versa. PSNR is often expressed on a logarithmic scale in decibels (dB). PSNR values falling below $30 \mathrm{~dB}$ indicate a fairly low quality, i.e. distortion caused by embedding can be obvious; however, a high quality stego-image should strive for $40 \mathrm{~dB}$ and above [28]. Our results indicate that embedding process introduces less perceptual distortion and higher PSNR [29]. Note that PSNR ranging from $40 \mathrm{~dB}$ to $45 \mathrm{~dB}$ means that the quality degradations could hardly be perceived by a human eye.

\section{Conclusion}

The proposed technique has its place in secured data communication. In this paper an attempt has been made to identify the requirements of a good data hiding algorithm. Hiding a message with steganographic methods reduces the chance of a message being detected. Steganography is the data hiding technique which comes under the assumption that if the feature is visible, the point of attack is evident, thus the goal here is always to obscure the very existence of the embedded data. Neither Steganography nor cryptography alone is a good solution for data secrecy from the attacks. But if these methods are combined, the system may provide more security to the data. If a message is encrypted and hidden with a steganographic method, it provides an additional layer of protection and reduces the chance of the hidden message being detected. This combinational methodology gives synergistic effect and will satisfy the requirements such as capacity, security and robustness for secured data transmission over an open channel. This paper mainly focuses on the development of a new system with extra security features where a meaningful piece of text message can be hidden by combining techniques like Cryptography and Steganography. These combined techniques can be propelled to the forefront of the current security techniques by the remarkable growth in computational power, the increase in security awareness among the individuals, groups, agencies, government organization and through intellectual pursuit. Here we embed the confidential message into an image file in such a manner that the degradation in quality of the carrier image is not noticeable. Thus the proposed method allows users to send data through the network in a secured fashion. The steganography method may be further secured if we compress the secret message first and then encrypt it and then finally embed inside in the cover image.

\section{ACKNOWLEDGEMENTS}

One of the authors (Shamim Ahmed Laskar) gratefully acknowledges UGC for the granting Research fellowship (Maulana Azad National Fellowship).

\section{REFERENCES}

[1] M. Conway, “ Code Wars: Steganography, Signals Intelligence, and Terrorism”, Knowledge Technology \& Policy, Volume 16, Number 2, pp. 45-62, Springer, 2003.

[2] R. J. Anderson and F. A. P. Petitcolas, "On The Limits of Steganography", IEEE Journal of Selected Areas in Communications, 16(4), pp.474-481, May 1998, ISSN 0733-8716.

[3] F. A. P. Petitcolas, R. J. Anderson and M. G. Kuhn, "Information Hiding-A Survey", Proceedings of the IEEE, 87(7), pp.1062-1078, July 1999. 
[4] S. A. Laskar and K. Hemachandran, "An Analysis of Steganography and Steganalysis Techniques", Assam University Journal of Science and Technology, Vol.9, No.II, pp.83-103, January, 2012, ISSN: 0975-2773.

[5] C. Hosmer, "Discovering Hidden Evidence”, Taylor \& Francis Group, Journal of Digital Forensic Practice, Vol. No.1, pp.47-56, 2006.

[6] B. Li, J. He, J. Huang and Y. Q. Shi, "A Survey on Image Steganography and Steganalysis", Journal of Information Hiding and Multimedia Signal Processing, Vol. 2, No. 2, pp. 142-172, April, 2011, ISSN 2073-4212.

[7] N.F. Johnson and S. Jajodia, "Exploring Steganography: Seeing the Unseen", IEEE, Computer, vol. 31, no. 2, pp. 26-34, Feb. 1998.

[8] A. J. Raphael and V. Sundaram, "Cryptography and Steganography - A Survey", Int. J. Comp. Tech. Appl., Vol 2 (3), pp. 626-630, ISSN:2229-6093.

[9] S. Song, J. Zhang, X. Liao, J. Du and Q. Wen, “A Novel Secure Communication Protocol Combining Steganography and Cryptography”, Elsevier Inc, Advanced in Control Engineering and Information Science, Vol. 15, pp. 2767 - 2772, 2011.

[10] M. A. Fadhil, “A Novel Steganography-Cryptography System”, Proceedings of the World Congress on Engineering and Computer Science 2010, USA, Vol. I, October, 2010, ISSN: 2078-0966.

[11] R. Anderson, "Cryptanalytic Properties of Short Substitution Ciphers", Taylor \& Francis, Cryptologia, Vol. XIII, No. 1, pp. 61-72, January, 1989.

[12] G. J. Simmons, "Subliminal Channels: Past and Present," European Transactions on Telecommunications, Vol. 4, No. 4, pp. 459-473, Aug 1994.

[13] R. S. Ramesh , G. Athithan and K. Thiruvengadam, "An Automated Approach to Solve Simple Substitution Ciphers", Taylor \& Francis, Cryptologia, Vol. XVII, No. 2, pp. 202-218, April, 1993.

[14] E. Walia, P. Jain and Navdeep, “An Analysis of LSB \& DCT based Steganography”, Global Journal of Computer Science and Technology, Vol. 10 Issue 1 (Ver 1.0), pp 4-8, April,2010.

[15] M. Kaur, S. Gupta, P. S. Sandhu and J. Kaur, "A Dynamic RGB Intensity Based Steganography Scheme”, World Academy of Science, Engineering and Technology 67, pp 833-836, 2010.

[16] P. Khare, J. Singh and M. Tiwari, "Digital Image Steganography", Journal of Engineering Research and Studies, Vol. II, Issue III, pp. 101-104, July-September,2011, ISSN:0976-7916.

[17] A. B Watson, "Image Compression Using the Discrete Cosine Transform", Mathematica Journal, 4(1), pp. 81-88, 1994.

[18] C-L Liu and S-R. Liao, "High-performance JPEG steganography using complementary embedding strategy", Elsevier Inc, Journal of Pattern Recognition Vol. 41, pp.2945 - 2955,2008.

[19] B. B. Zaidan, A. A. Zaidan, A.K. Al-Frajat and H.A. Jalab, "On the Differences between Hiding Information and Cryptography Techniques: An Overview", Journal of Applied Sciences, Vol.10, No.15, pp.1650-1655, 2010.

[20] M. Kharrazi, H. T. Sencar and N. Memon, "Performance study of common image steganography and steganalysis techniques”, Journal of Electronic Imaging, SPIE Proceedings Vol. 5681.15(4), 041104 (Oct-Dec 2006). SPIE and IS\&T., 2006.

[21] B.J. Erickson, "Irreversible Compression of Medical Images", Journal of Digital Imaging, Vol. 15, No.1, pp. 5-14, March, 2002.

[22] A. B. Watson, "Perceptual Optimization of DCT Color Quantization Matrices", Proceedings of the IEEE International Conference on Image Processing, Austin, TX, Nov., 1994.

[23] X. Li and J. Wang, "A steganographic method based upon JPEG and particle swarm optimization algorithm”, Information Sciences 177 (15) (2007) 3099-31091.

[24] N. Provos and P. Honeyman, "Hide and seek: An introduction to steganography", IEEE Security and Privacy1(3) pp. 32-44, 2003.

[25] S. Ravi and K. Knight, “Attacking Letter Substitution Ciphers with Integer Programming”, Taylor \& Francis, Cryptologia, Vol.33, No.4, pp.321-334, 2009.

[26] P. Kaijser, T. Parker, and D. Pinkas, "SESAME: The Solution to Security for Open Distributed Systems," Journal of Computer Communications, Vol. 17, No. 4, pp. 501-518, Jul 1994.

[27] G.W. Hart, "To Decode Short Cryptograms," Communications of the ACM, Vol. 37, No. 9, pp. 102108, Sept 1994. 
[28] B. E. Carvajal-Gámez , F. J. Gallegos-Funes and J. L. López-Bonilla, " Scaling Factor for RGB Images to Steganography Applications", Journal of Vectorial Relativity, Vol.4, No.3 pp.55-65, 2009.

[29] G. Ulutas , M. Ulutas and V. Nabiyev, "Distortion free geometry based secret image sharing", Elsevier Inc, Procedia Computer Science, Vol.3, pp.721-726, 2011.

[30] W.F. Friedman, "Cryptology," Encyclopedia Britannica, Vol. 6, pp. 844-851, 1967.

[31] Atul Kahate, "Cryptography and Network Security", 2nd Edition, Tata McGraw-Hill, 2008.

[32] R. C. Gonzalez and R. E. Woods, "Digital Image Processing", 2nd edition, Prentice Hall, Inc, 2002.

\section{Authors}

Shamim Ahmed Laskar received his B.Sc. and M.Sc. degrees in Computer Science in 2006 and 2008 respectively from Assam University, Silchar, where he is currently doing his Ph.D. His research interest includes Image Processing, Steganography, Information Retrieval and Data Security.

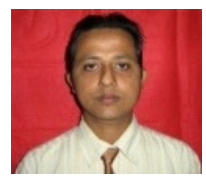

Prof. K. Hemachandran is associated with the Department of Computer Science, Assam University, Silchar, since 1998. He obtained his M.Sc. Degree from Sri Venkateswara University, Tirupati and M.Tech and Ph.D Degrees from Indian School of Mines, Dhanbad. His areas of research interest are Image Processing, Software Engineering and Distributed Computing.

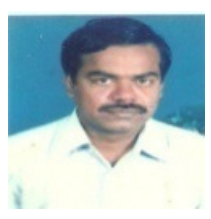

